\title{
Incorporating SCADA modules into Introductory Programmable Logic Con- troller Curriculum
}

\section{Prof. Christopher John Akelian, Cuesta College}

Education: 1996 MSEE San Diego State University 1987 BSEE Cal Poly San Luis Obispo

Work History: - Full time Engineering and Technology Instructor since 1997 (Co-PI on the NSF CREATE grant the entire time) - Digital Design Engineer 1987 to 1997 at Hughes Network Systems and General Instruments in San Diego - Sonar Technician with the Navy and Electronics/Networking Warrant Officer with the Coast Guard Reserves and currently in the Army National Guard (38 years total service with 6 years active duty and 32 years in reserves) 


\section{Incorporating SCADA Modules into Introductory Programmable Logic Controller Curriculum}

\section{Introduction}

This paper will present some of the challenges of developing SCADA(Supervisory Control and Data Acquisition) curriculum for a community college Programmable Logic Controller course. It will discuss the research of industrial products and choices made to provide an inexpensive solution to offer training on this complex technology. It will also show some of the simple but effective test fixtures and software interfaces that were developed to provide modules of lab work that give students an overview of the technology in as little as six to eight 3 hour lab sessions.

Introducing SCADA training into our community college Programmable Logic Controller curriculum proved to be a difficult task. Having no SCADA background it took several hundred hours of research and development to create training equipment and put together a set of basic instruction modules. The motivation to start this journey came from my role in the NSF grant called CREATE. CREATE an Advanced Technology Education grant that evolved several times since its inception in 1997 took on the task of developing Alternative Energy Curriculum and training in 2009. My proposal in the grant was to develop curriculum that tied alternative energy monitoring concepts to our Programmable Logic Controller course using SCADA systems.

Starting the project my only knowledge of SCADA systems was understanding their importance in monitoring and controlling industrial processes - and that they were intimately tied into Programmable Logic Controllers (PLCs) to make the systems function. Students that worked at utility plants in particular would take our PLC course and would frequently ask questions regarding the role of PLCs in SCADA systems. Unable to adequately answer those questions was convincing evidence of the need to incorporate SCADA concepts into our course. Our community college being in a rural area with a limited number of students prevented us offering an entire course in just SCADA systems; therefore, adding modules within our existing course was our only option.

The original proposal was based on monitoring an outdoor solar field and transmitting the sensor data back into our lab wirelessly from about a half mile away. This changed over time to make the training more accessible inside the classroom; therefore, a totally indoor solution was developed including an inverter that could run on 4 small solar panels mounted on a portable rolling unit that also had the optional capability to be powered from a 120VDC power supply connected to the wall outlets inside the lab. Eventually it became apparent that to give the SCADA training all that was needed was a desktop power supply and a small load box that simulated the solar array for outputs into the monitoring sensors. 
As far as SCADA training materials availability, very little exists that is directed toward community colleges. Most of the training materials are specific to vendor equipment and is tailored toward working engineers in short courses offered by the vendor. Therefore, the training materials for this project were developed around the specific equipment we were using in our lab. Also, the SCADA software selected was chosen to make the implementation of the training modules as low cost and sharable as possible. As the project progressed the SCADA training materials evolved into about 8 three-hour sessions of lab based-training. The training steps the students through the basics of creating a PLC program for SCADA applications, configuring communications software to connect to a SCADA package, and finally configuring and programming a SCADA human machine interface.

The background needed to hold the training was a solid understanding of the PLC wiring and programming, which constitutes $75 \%$ of the training in our PLC course. The basic programming background is an understanding of digital logic and its application to PLC Ladder logic. Also, the understanding of how signals are input and output to and from the PLC and are referenced in the PLC memory register sets. Further, an understanding of the PLC communications both serial and Networking are essential background needed to understand the SCADA software and how it interacts with the PLC.

\section{Finding a SCADA software solution}

In searching the internet for various SCADA solutions a company on the Gulf Coast of Louisiana called NSI Upstream offered to give us their Oil Field Commander SCADA software for classroom use. It required a server and was licensed to us for 10 client stations. At the time it proved impossible to work with our existing Allen Bradly SLC500 PLC equipment since it needed a MODBUS capable protocol interface to communicate over. And on top of this there were several hundred pages of nearly incomprehensible documentation in the form of computer help files so other options were pursued.

Allen Bradley FactoryTalk View was investigated which is somewhat of an industry standard but the pricing and complexity was an obstacle - particularly since one of the objectives of the grant was to create training that was easily shared with other schools. Several other low cost options were investigated - finally a well-recognized industrial package that had a free version (from a company called IGSS which is now Schneider Electric) was selected. It has a free version called IGSSFREE50, which has all the capability needed to hold training and teach SCADA concepts. Plus it has online training materials and videos to help learn how to use the product. This product has a stand-alone workstation installation not requiring a separate server but has a local database to record data; thus minimizing the requirements for sharing the training.

The next hurdle in this process was to attempt connectivity to our Allen Bradley Micrologix 1000 and SLC 500 series of PLCs. It became apparent there was no way to directly connect these models due to the lack of TCP/IP communications and due to the old serial protocol DF1 
that they utilized. The option for our situation was either upgrade the PLCs by purchasing a 1761-NET-ENI adapter to support TCP/IP, or purchasing (at a minimum) the Micrologix 1100 series PLC that had the TCP/IP interface built-in and also supported the popular industrial network protocol called MODBUS. We ended up doing both, which proved to be a good choice.

Once the TCP/IP communication capability was added it became apparent that the IGSS SCADA communication drivers still could not talk directly to the Allen Bradley products since Allen Bradley still encapsulated its DF1 protocol into TCP/IP. The next discovery was it required a middleman to translate the Allen Bradley TCP/IP-DF1 format to a standard format understood by all SCADA systems called OPC (OLE for Process Control). The SCADA system had an OPC driver that passed information over TCP/IP as an OPC Client. Therefore, an OPC Server was required to make the translation between the Allen Bradley TCP/IP-DF1 to TCP/IPOPC so that the PLC could communicate to the SCADA OPC client configured system.

Allen Bradley has a product called RXLinx that can act as an OPC server. There is a free version called RXLinx Classic Lite, which is used to establish communications from their PLC Programming software called RSLogix and the lower end Allen Bradley products we use in our training program such as the Micrologix and SLC 500 series controllers. Unfortunately, the free version does not support the OPC server function and the versions that do cost over $\$ 1000$. After searching online two main OPC server software companies were available (Matrikon and Kepware); they both supported Allen Bradley PLCs and had evaluation versions (both have a two-hour run time in evaluation mode before restarting the OPC Server service for another two hours).

Matrikon was chosen since it appeared easier to configure; however, the auto-configure feature had many problems for our exact models that caused more time and effort to resolve than any other aspect of the project. Once these problems were resolved configuring the SCADA software to the OPC server and creating a simple display were relatively easy.

The total software solution to implement the SCADA training (for either a Micrologix 1000 or 1100 series PLC's) using all free software included: Allen Bradley Micro_Lite 8.3 (this is the free version of the RSLogix 500 programming environment); RSLinx Classic Lite vs. 2.57; IGSSFree50 vs. 10 (version 11 was also tested), and Matrikon OPC vs. 3.01 for Allen Bradley PLC's.

\section{SCADA hardware training solutions}

Part of the training solution was previously described in the introduction. The simple load box and power supply was all that was needed to simulate a solar array and to hold introductory training on implementing power measurements and displaying what could be a solar array voltage, current and power. Along with the solar array simulation the proper sensors and Micrologix PLC on a simple training board were developed. 
Other training developed related to Alternative Energy monitoring was based on weather monitoring needed to verify that the solar panels are operating at full efficiency. The weather station equipment had sensors such as Radiance, UV, Wind direction and speed and Panel temperature (many other sensors are available). Ambientweather weather station kits were chosen that had a MODBUS Remote Terminal Unit (RTU) and also a MODBUS TCP gateway to expose the students to industrial protocols. Simple fixtures were made using common PVC pipe as platforms to mount the sensors. A Micrologix 1100 PLC training board was developed to collect the weather station data via the Ocean Controls MODBUS controller (the Weather Station MODBUS controller is part of the weather station kit) and communicated using an Allen Bradley 1763-NC01 cable.

Another piece of equipment was developed to fill a gap since the power and weather monitoring only read sensors but do not send any control signals to control them. A small Motor control trainer was designed to provide the solution for this limitation. The motor trainer allowed for training in such topics as process control of reciprocating operations and designing a SCADA control panel to control and monitor the operations.

Finally a PLC-free solution was developed to provide training that only required a Weather Station and an Ocean Controls MODBUS Gateway. This reduced the level of student understanding needed to offer the training so that possibly a High School program could offer portions of it.

\section{Student learning outcomes}

The student learning outcomes of the SCADA training developed evolved over time since many of the early objectives were revised to shorten the length of the training. The original objectives for the training included wiring and configuring wireless access of the portable solar-arrayinverter training platform. After offering the training in the PLC course for the first time it was clear that these objectives were taking away from the time needed to teach PLC programming concepts. Several of the objectives were transferred to other courses so that the emphasis of the training in the PLC course could focus on SCADA concepts alone. The following are the SCADA related student learning outcomes within our one semester PLC course:

- Identify the role of SCADA systems in industry, the basic components of a SCADA system, and how the PLC is used in SCADA solutions

- Wire a SCADA capable PLC using both sensors and actuators for solar monitoring and motor control applications

- Program a PLC to collect data for a SCADA system using both Analog and Discrete sensors

- Identify various software packages needed to communicate in a SCADA system

- Configure and test communications in a SCADA system

- Create a Human Machine Interface (HMI) that reflects the operation of equipment 
- Troubleshoot problems that occur while integrating a SCADA system

\section{Student activities and modules developed}

Introducing the SCADA curriculum for the first semester was an extreme challenge since the lab modules were untested and it wasn't clear how much of the curriculum was needed to effectively give the students an understanding of SCADA concepts. Through trial and error over the last 4 course offerings the curriculum was reduced to about five weeks (of two 3 hour sessions per week) worth of essential presentations and lab modules. The following is a breakdown of the training:

- Week1: General SCADA introduction and labs in identifying and wiring SCADA compatible hardware including PLCs, power sources, sensors and actuators.

- Week2: Discuss SCADA communication concepts, including protocols and network concepts needed to understand how the system links together. Perform labs in configuring network IDs into the PLCs and network adapters in a LAN setting.

- Week3: Introduce Analog to Digital conversion, and how the PLC must compute floating point and integer number results using scaling to format the data for SCADA software packages. Perform power computations needed to process solar panel voltage and current outputs. Perform labs in PLC program creation for power monitoring, then pass the data to SCADA software packages for intermediate processing and display.

- Week4: Introduce weather station hardware and sensors. Discuss the MODBUS industrial network protocol and PLC messaging needed to interface a Remote Terminal Unit (RTU) to the PLC. Perform labs in RTU configuration and PLC messaging for MODBUS data collection, then pass the data to SCADA software packages for intermediate processing and display.

- Week5: Introduce discrete control concepts in the SCADA systems. Adapt previous motor control experiments to SCADA applications. Perform labs in creating Human Machine Interfaces for discrete control. Integrate motor control hardware and program the PLC for remote control processing by SCADA software.

\section{Assessment of curriculum effectiveness}

The effectiveness of the curriculum was assessed using several modes: lab work results, tests and practicals, and student surveys for feedback on their understanding of the training. Below is a summary of the results. 
- Lab work results: over the course of 4 class offerings the completion of the lab work improved with each revision of the modules. In the last class the students had an $89.8 \%$ completion rate of all the SCADA related labs.

- The written test results: over the course of the class offerings the test results were mixed. In the $4^{\text {th }}$ class offering the students showed a $73.3 \%$ average on the SCADA related concepts; however, $61 \%$ of the class were $79 \%$ and above and $30.8 \%$ were below $60 \%$ showing that concepts were well understood by most of the class but some of the students severely struggled.

- Student survey results: the results of the of the student surveys regarding their perceived comprehension of SCADA concepts improved dramatically over four separate course offerings. The first course offering only $63.2 \%$ felt they understood the SCADA concepts as opposed to the $4^{\text {th }}$ course offering where $95.4 \%$ responded that they believed they fully understood the topics presented. This improvement was most likely due to the revised lab content and streamlining the training; however, based on the test results they probably were overconfident due to getting the SCADA labs functioning as opposed to the actual theory of what they were performing. Also; based on written feedback, some of the students currently working in industry wished that the training was created around a more recognized SCADA package such as Wonderware or FactoryTalk but felt the training was still very useful to understand the basic concepts.

\section{Conclusions}

Introducing SCADA training into an existing one semester PLC course was challenging and filled with many technical and pedagogical obstacles. Although, the training has shown to be effective in introducing SCADA concepts it and has taken away from some of the basic PLC programming tasks that were previously taught. On one hand the students have shown to be better-rounded having a broad overview of the PLC and its related applications but lacked some programming skills to make them effective programmers. In our case, I believe this tradeoff is more beneficial for student success since it was very difficult to make PLC programmers out of students in only one semester; therefore, the broader scope including network communications and SCADA systems gives them more tools to present to employers.

\section{Instructor training outcomes}

The expected outcomes of this conference training are:

- Show how effective SCADA training modules can be integrated into a single PLC course

- Show how SCADA training can be introduced at a low cost

- Show how simple test fixtures and electro-mechanical devices can be constructed by students to deliver an overview of SCADA operations 
- Show how SCADA interfaces can be created by students in short lab sessions to provide effective training

- Supply the lab curriculum materials and plans, including parts lists and free software to reproduce the training

Below are some pictures of the training equipment, PLC programs, and SCADA displays developed in the Lab modules.

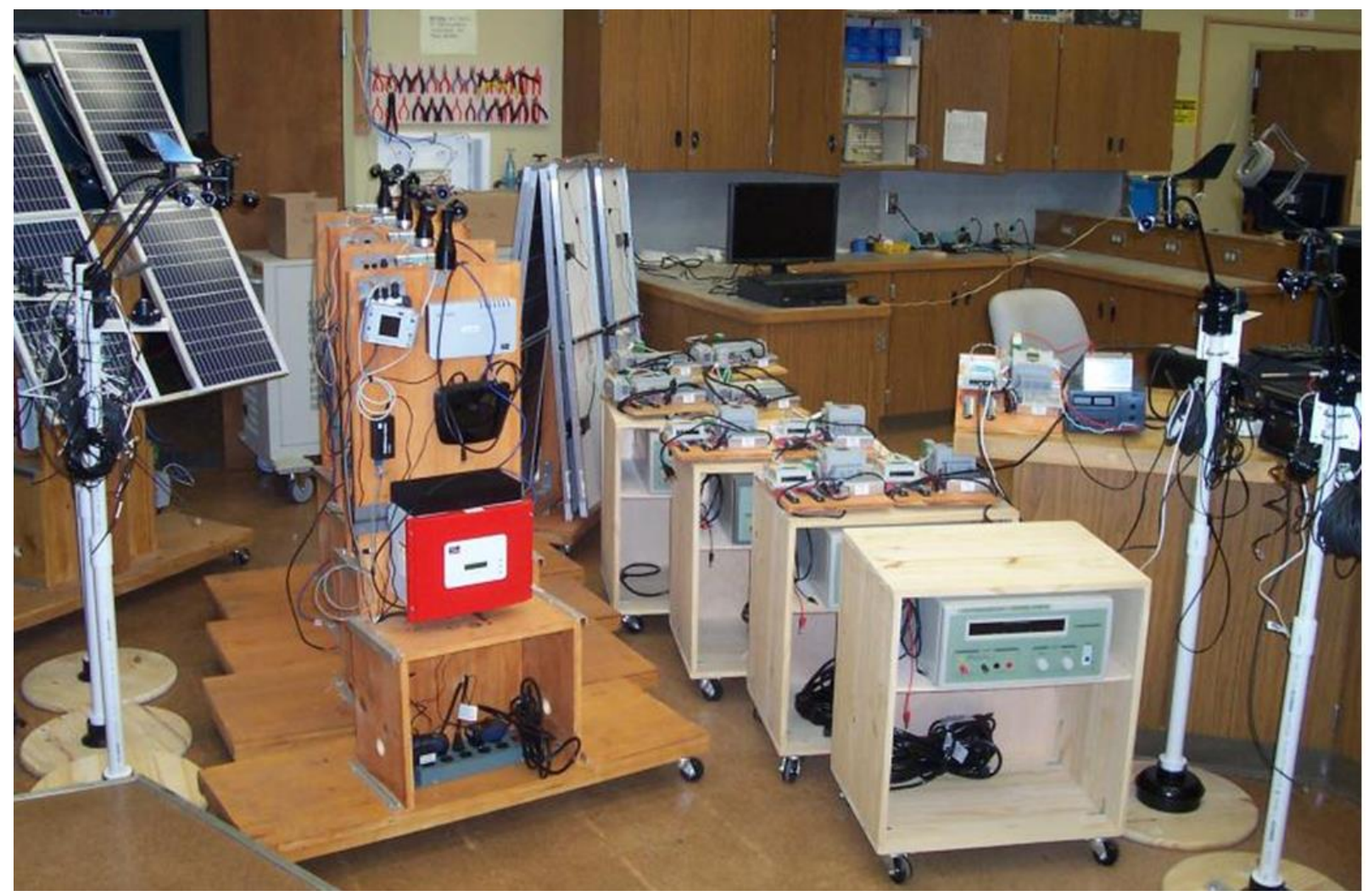

Figure 1 This picture shows some of the equipment developed in-house for alternative energy SCADA training. The large roller platform in the center housed an inverter and a weather station that was monitored wirelessly. On the side of the roller platform PLC training cards could be mounted for monitoring the inverter voltage and current and passing information to a SCADA system. 


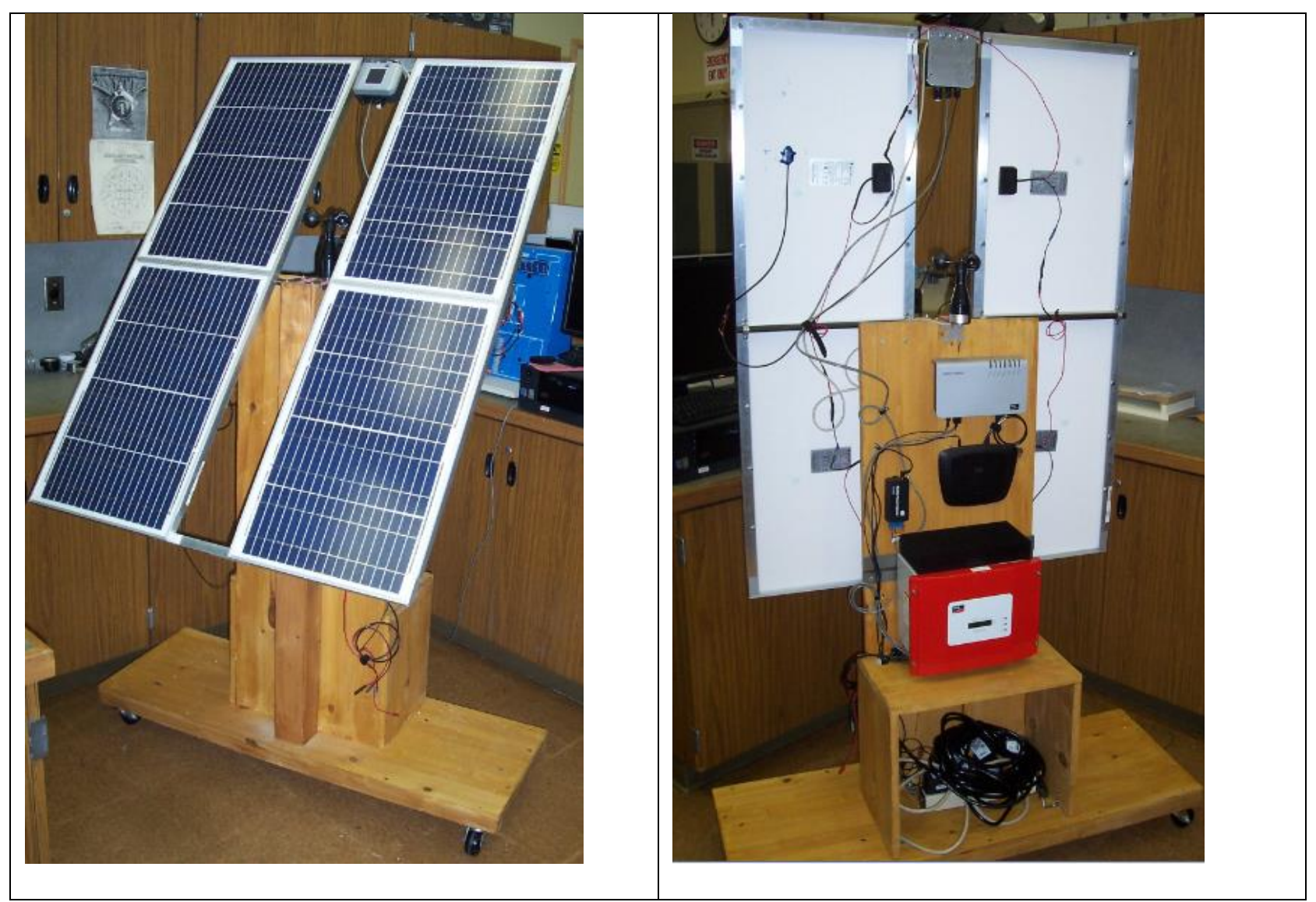

Figure 2 shows the solar inverter test fixture developed for solar monitoring

Power calculation simulation.

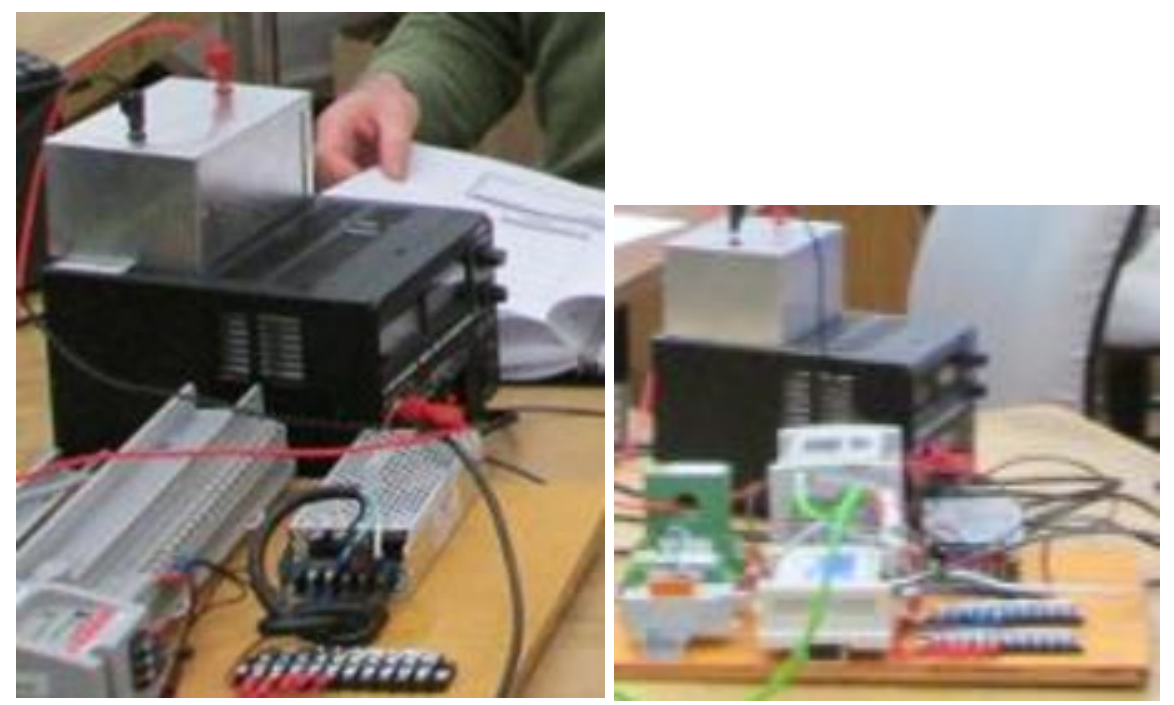

Figure 3 show how a small load box (this simulates the solar array of figure 2) is connected to either a Micrologix 1000 or a Micrologix 1100 using current and voltage sensors. 


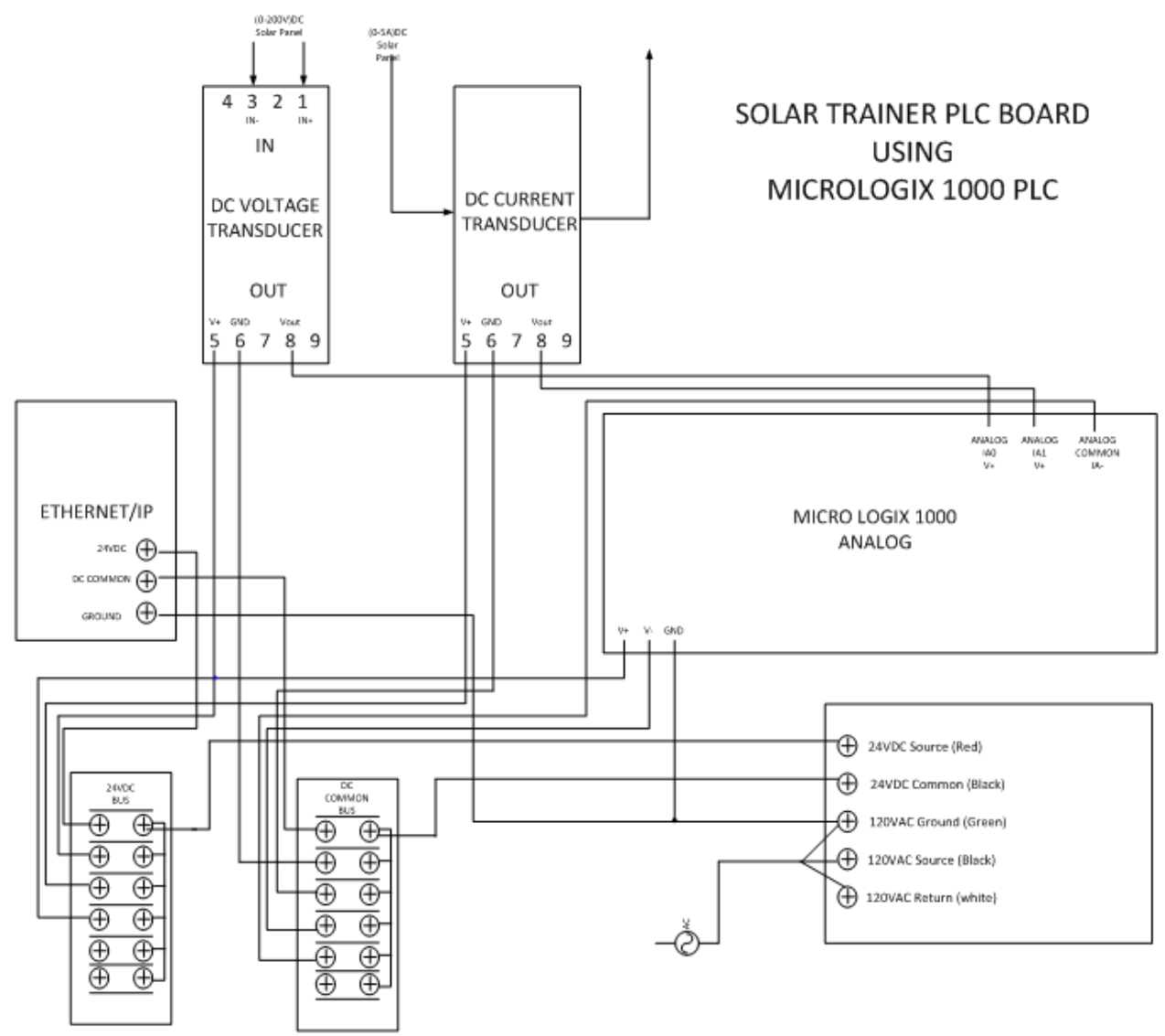

Figure 4 is an example wiring diagram for the PLC trainer board

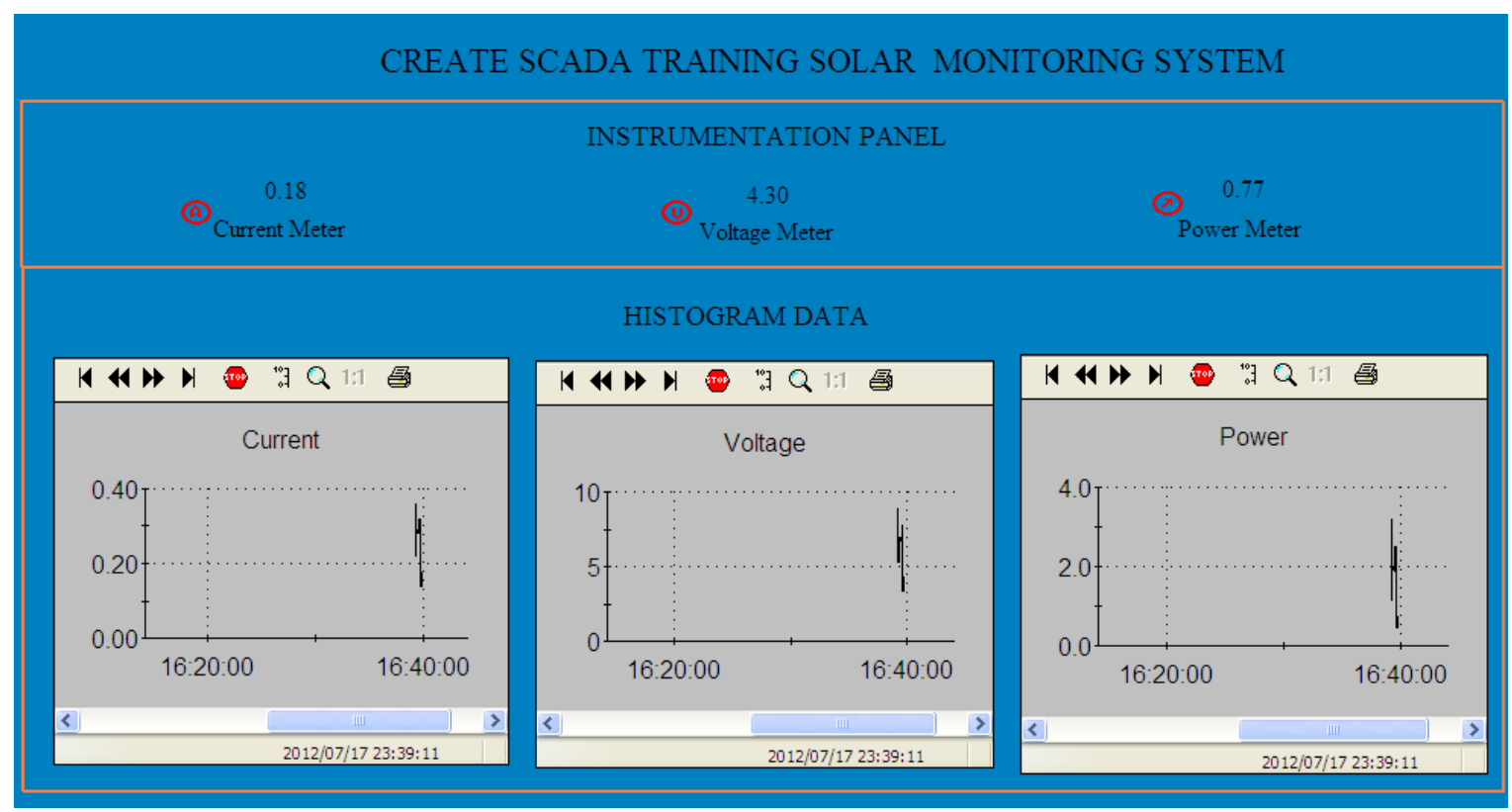

Figure 5 shows the SCADA interface created in the Lab module to monitor the solar array voltage, current, and power. 


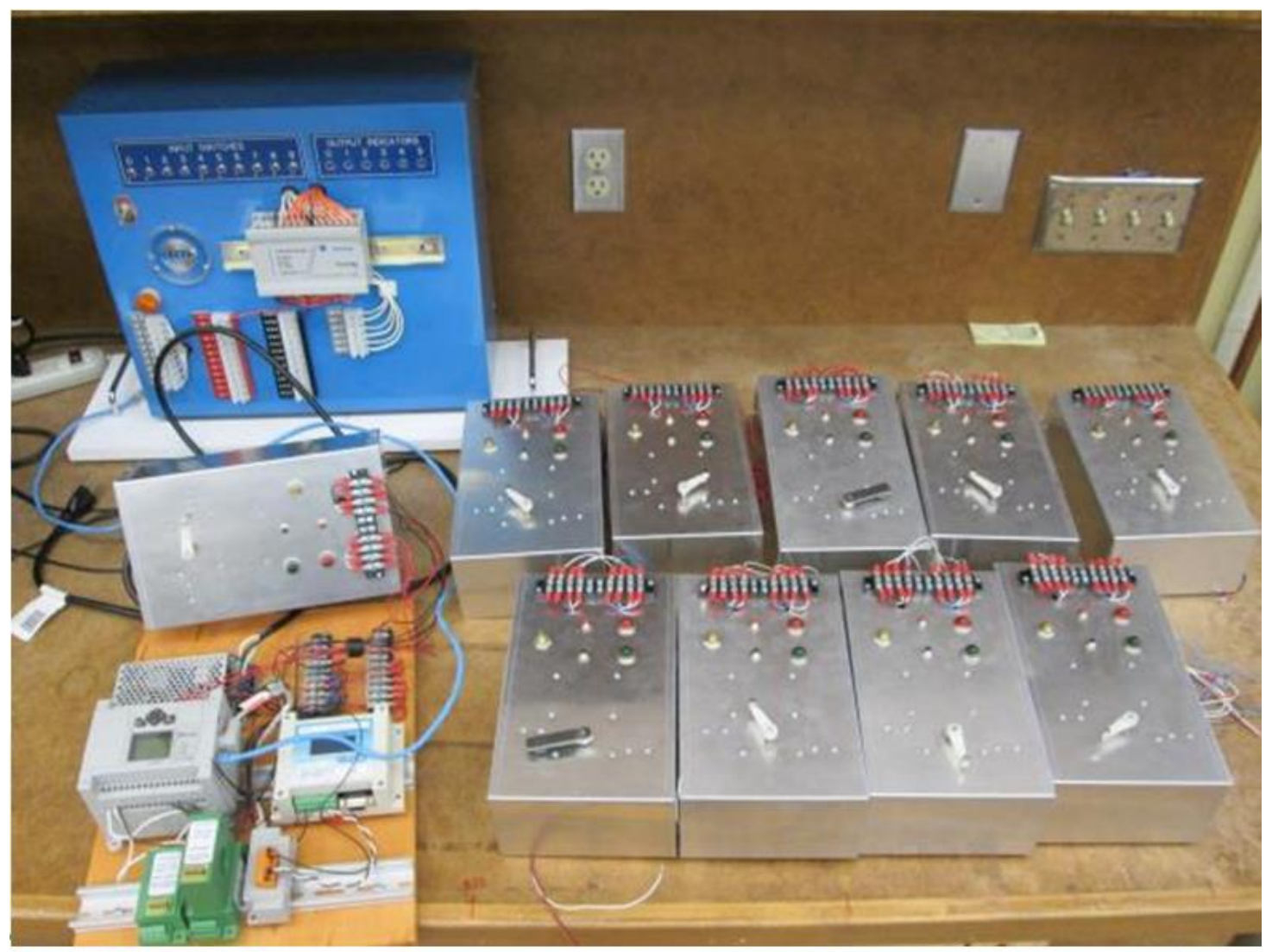

Figure 6 shows the in-house built Micrologix 1100 PLC board and Motor Control Boxes

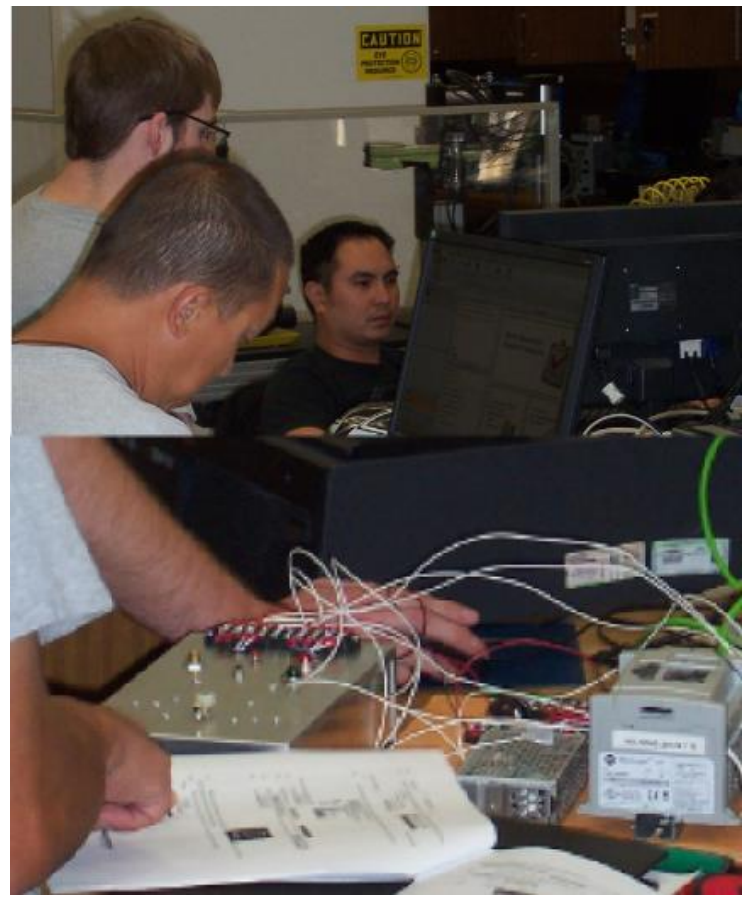

Figure 7 are students creating the PLC program and SCADA interface for the Motor control box.

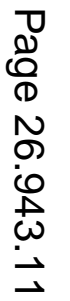




\section{MOTOR TRAINER BOX WIRING DIAGRAM}

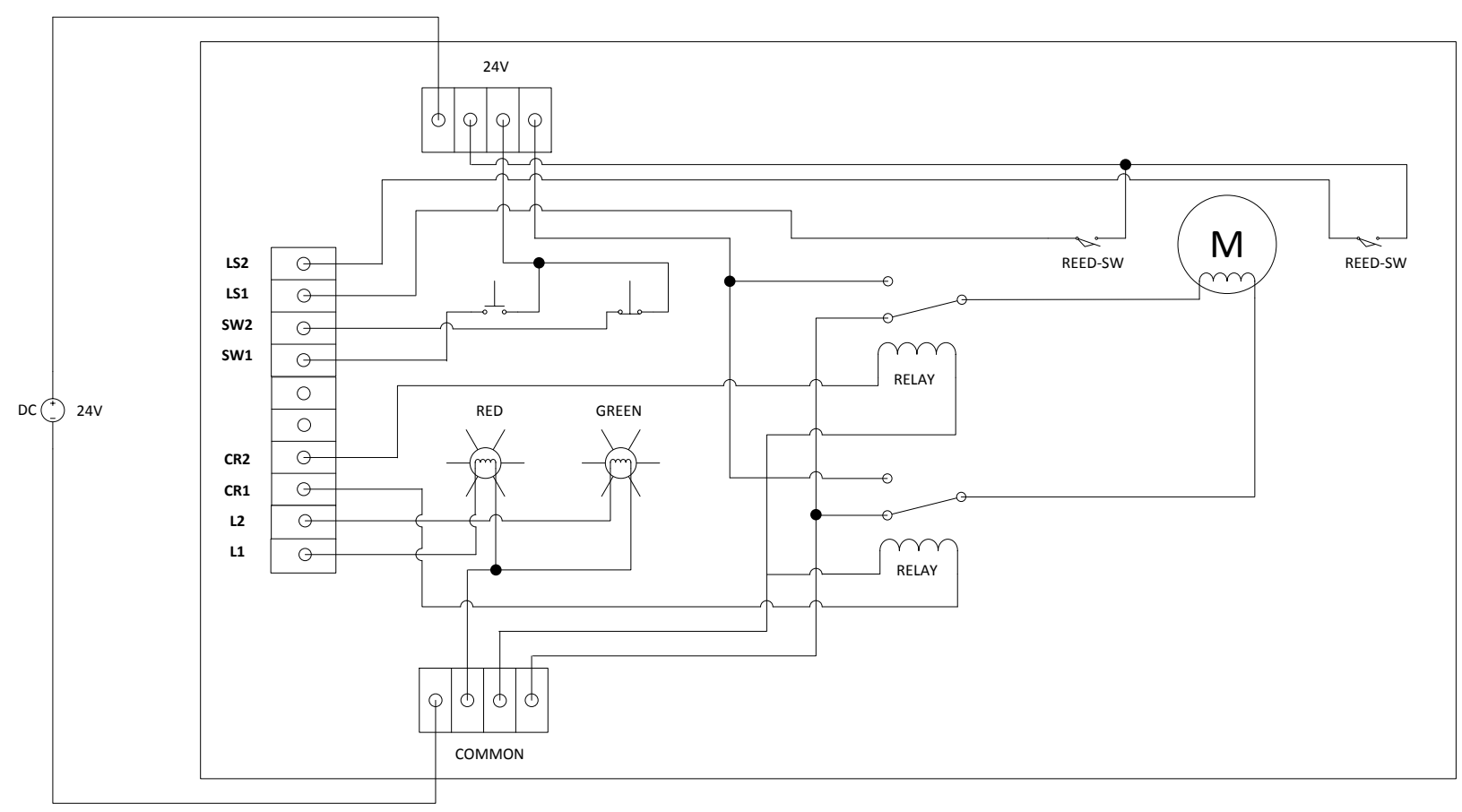

Figure 8 shows the internal wiring of the motor control trainer box
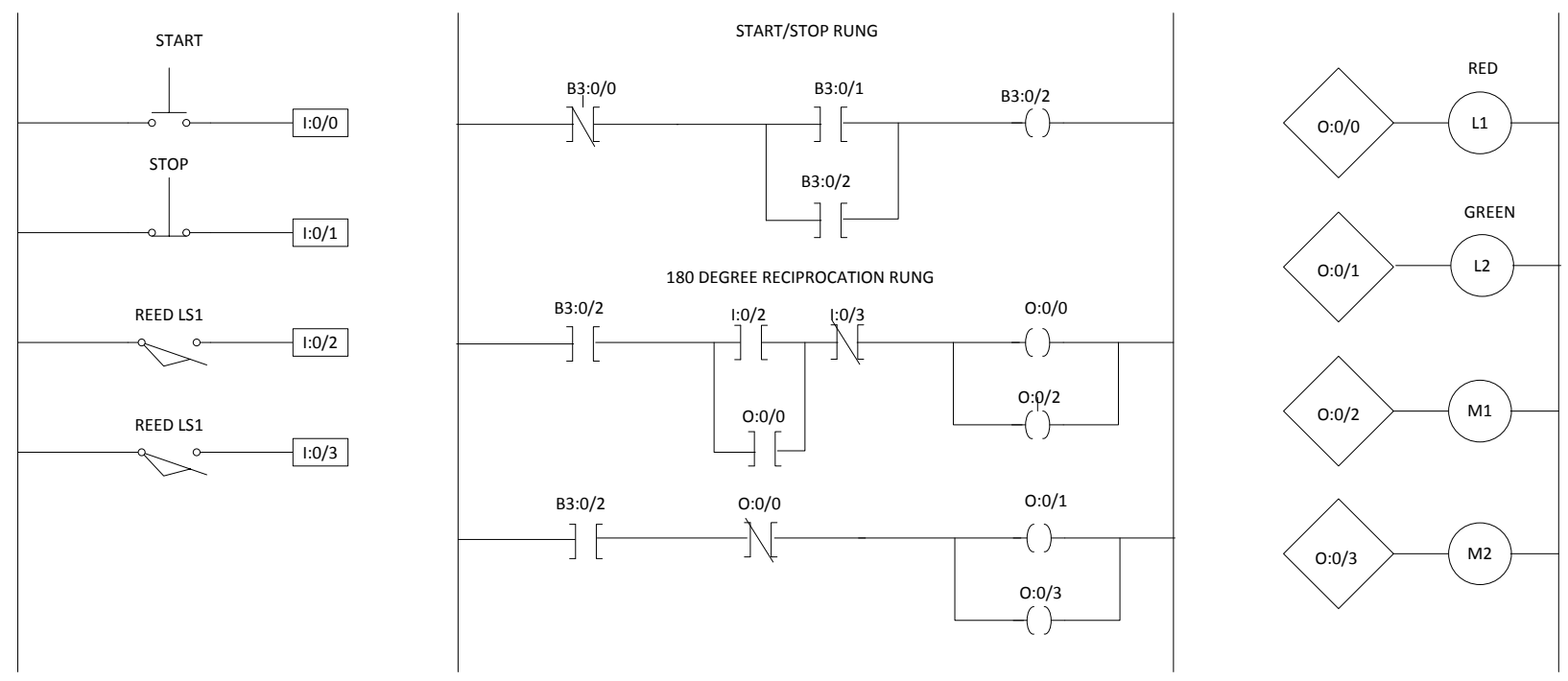

Figure 9 shows an example PLC program to run the motor control trainer for reciprocating operation 


\section{MOTOR CONTROL PANEL}

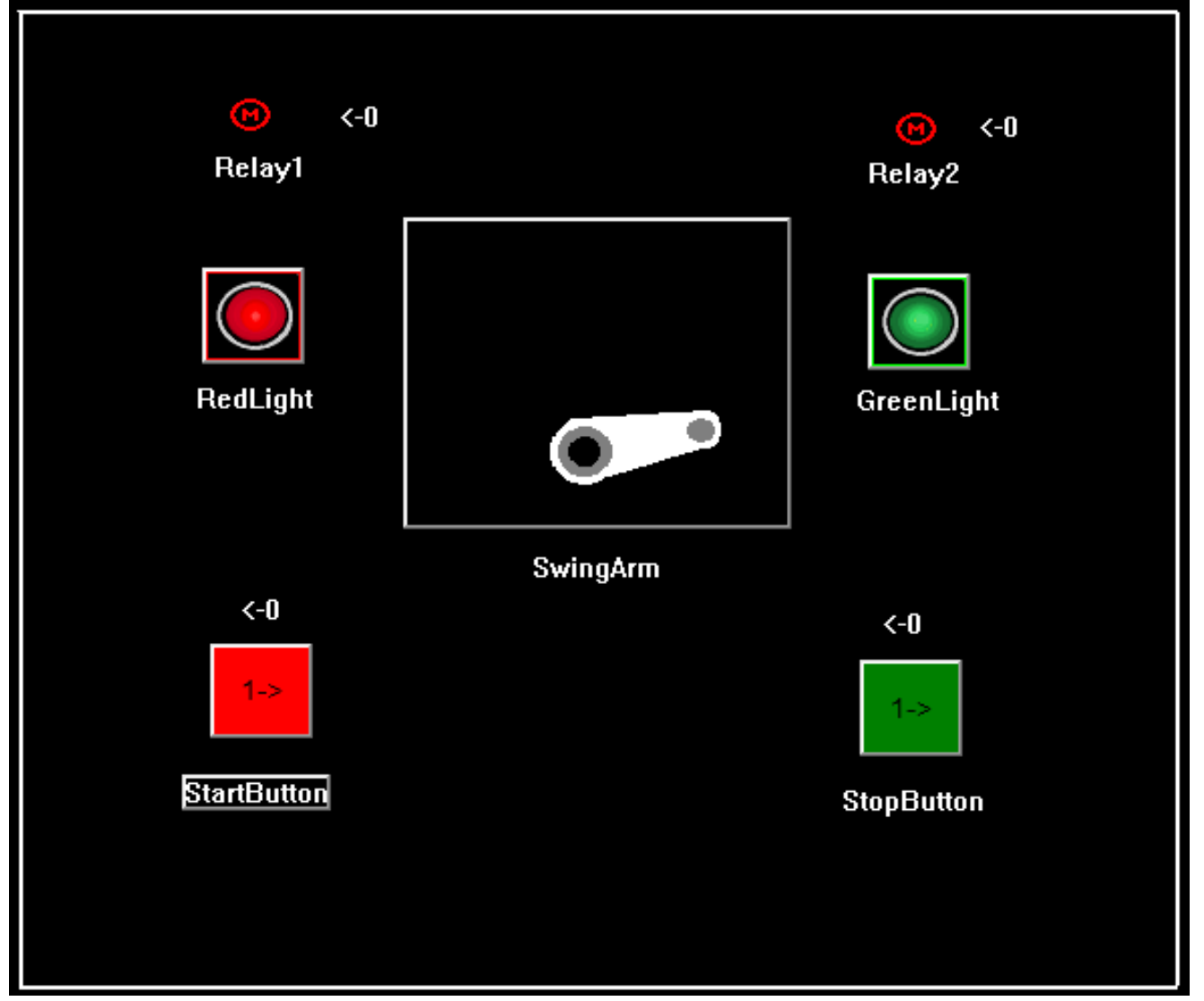

Figure 10 shows the SCADA display designed in the lab to operate the motor control trainer box

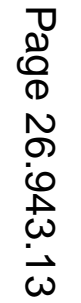




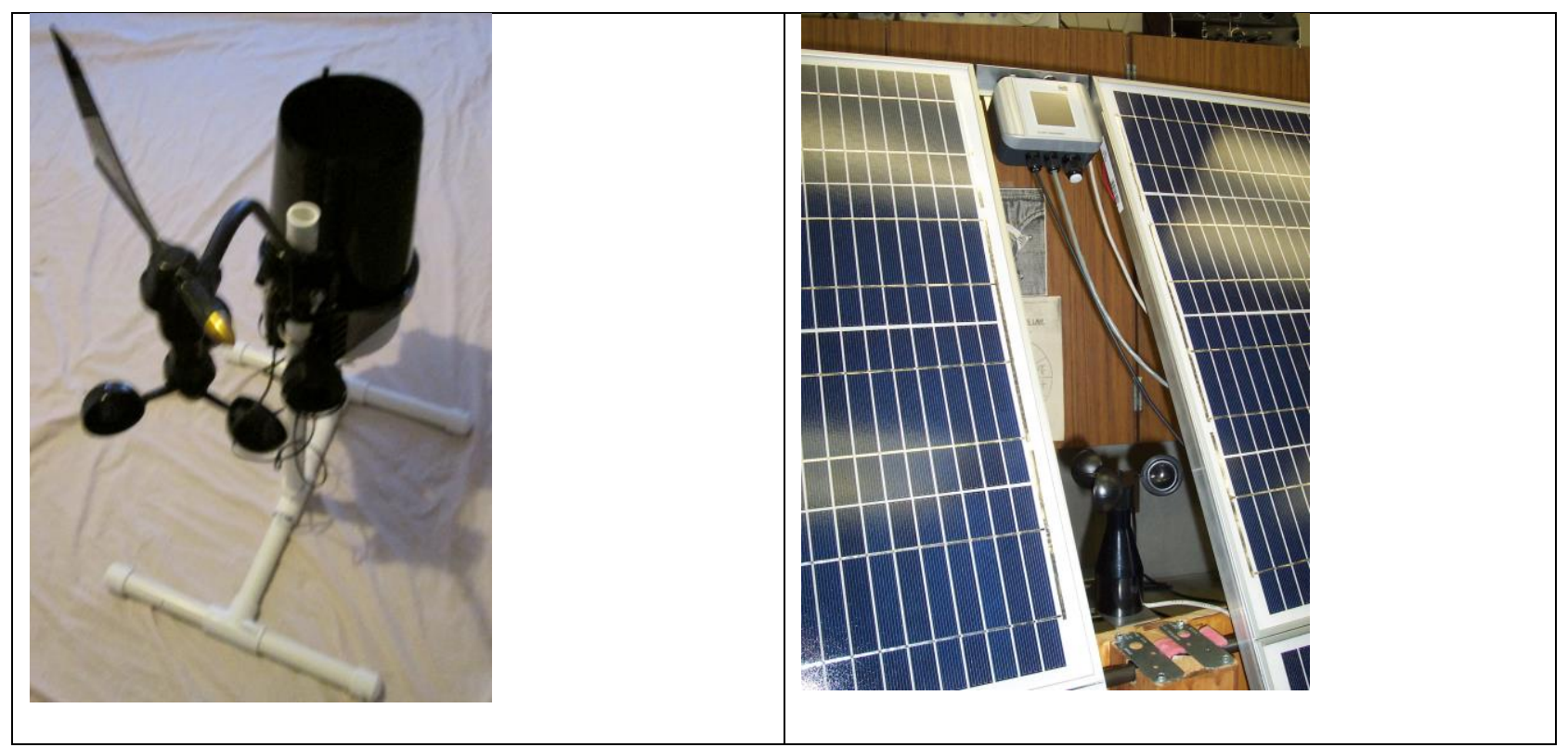

Figure 11 shows the weather monitoring sensors mounted on either an independent PVC platform or integrated into a Solar Array Inverter platform

\section{WEATHER DASHBOARD}

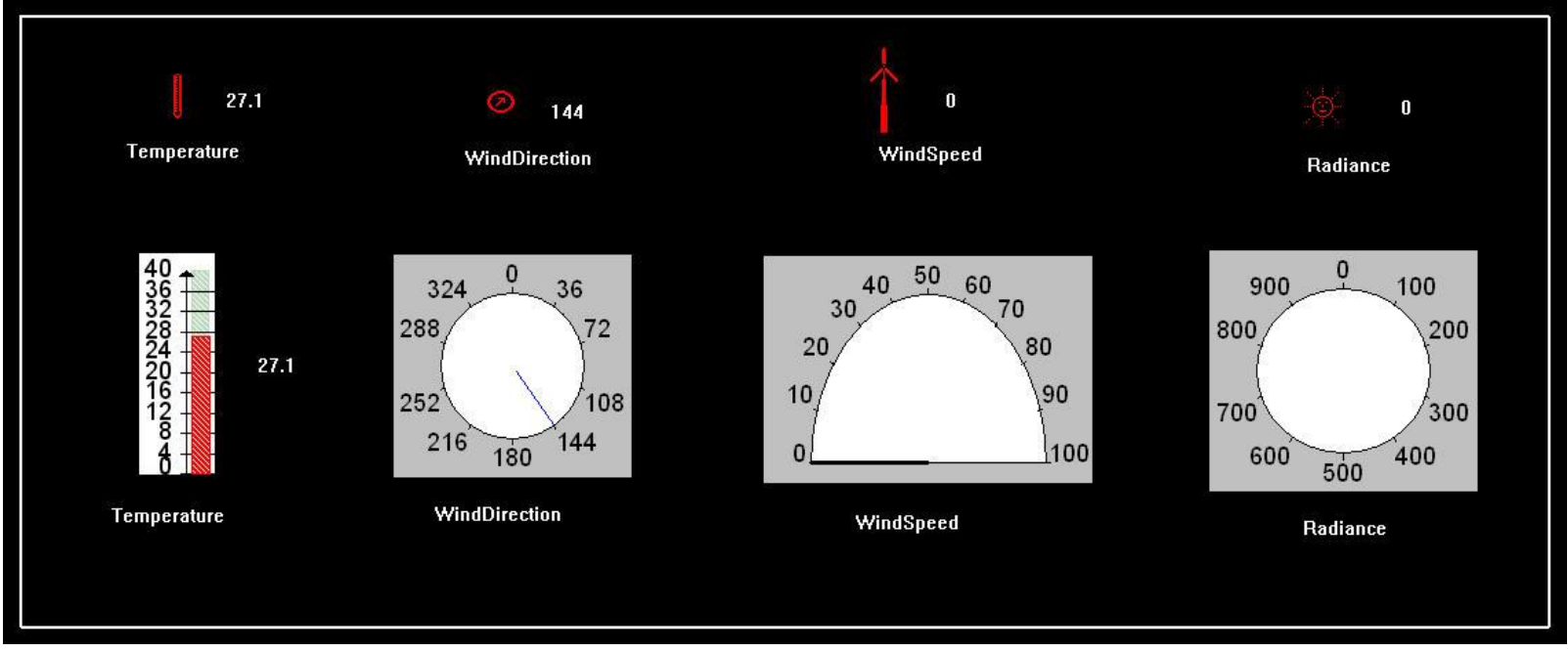

Figure 12 shows the SCADA interface to display the weather station data

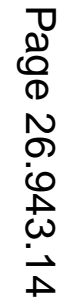

